\title{
Industrial Knowledge Graphs Workshop 2017
}

\author{
co-located with the 9th International ACM Web Science Conference 2017
}

\author{
Varish Mulwad, Raghava Mutharaju \\ GE Global Research \\ Niskayuna, NY, USA \\ (varish.mulwad,raghava.mutharaju)@ge.com
}

\begin{abstract}
This document provides a summary of the Industrial Knowledge Graph Workshop co-located with the ACM Web Science 2017 conference held on June 25, 2017, in Troy, NY, USA.

\section{ACM Reference Format:}

Varish Mulwad, Raghava Mutharaju . 2017. Industrial Knowledge Graphs Workshop 2017. In Proceedings of WebSci '17: ACM Web Science Conference CD-ROM, Troy, NY, USA, fune 25-28, 2017 (WebSci '17), 3 pages.
\end{abstract}

\section{PREFACE}

Search engines such as Google, Bing and intelligent assistants such as IBM Watson, Siri and Cortana have demonstrated tremendous benefits to users from consuming data from the knowledge graphs. There are several well-known knowledge graphs such as DBpedia, Wikidata and Schema.org in the consumer space, but very little attention has been given to knowledge graphs in non-consumer space. One such key area where more and more applications are making use of the structured data in the form of knowledge graphs is in the Industrial businesses such as manufacturing, oil and gas, power, aviation and mining. Industrial knowledge graphs can play an important role in creating Artificial Intelligence applications in the Industrial space. There is less focus on such Industrial knowledge graphs in the main track of research conferences.

A half day workshop, named "Industrial Knowledge Graphs" was organized on June 25, 2017, with the goal to bridge this gap and bring together researchers and practitioners in the areas of Web Science, knowledge graphs along with Industry subject matter experts. This was the first edition of the workshop and it was co-located with the 9th International ACM Web Science Conference 2017 in Troy, NY, USA. The call for papers encouraged to define and shape the vision of an Industrial knowledge graph with focus on subjects such as discussion on the nature of knowledge graphs and their applications in an Industrial setting. The workshop received three full paper and one short paper submission. Each paper was assigned to three reviewers from the program committee; all four submissions were accepted. The workshop was highlighted with a keynote talk by Prof. Craig Knoblock titled "Extracting, Aligning, and Linking Data to Build Knowledge Graphs" which presented the academic perspective on domain specific knowledge graphs. On the other

Permission to make digital or hard copies of part or all of this work for personal or classroom use is granted without fee provided that copies are not made or distributed for profit or commercial advantage and that copies bear this notice and the full citation on the first page. Copyrights for third-party components of this work must be honored.

For all other uses, contact the owner/author(s)

WebSci '17, fune 25-28, 2017, Troy, NY, USA

(c) 2017 Copyright held by the owner/author(s).

ACM ISBN 978-1-4503-4896-6/17/06.

https://doi.org/10.1145/3091478.3162382 hand, an invited talk from Arun Subramaniyan (presented by Mahadevan Balasubramaniam) titled "Leveraging Knowledge Graphs for Building Data-driven Products: An Oil \& Gas Perspective” provided the industrial perspective on this subject. The presentations at the workshop focused on several interesting domains such as the use of knowledge graphs to assist law enforcement agencies to stop human trafficking to its use to detect traffic events and in industrial production lines.

The workshop concluded with a panel discussion on "Industrial Knowledge Graphs: Opportunities and Challenges". The discussion was moderated by Justin McHugh (GE Global Research) and the panel members included Craig Knoblock (University of Southern California); Andrew Crapo (GE Global Research); Achille Fokoue (IBM T.J Watson); Evren Sirin (Stardog); Deborah McGuinness (Rensselaer Polytechnic Institute) and Peter Fox (Rensselaer Polytechnic Institute). We provide a brief summary of the panel discussion here. The discussion started with the question of "What is a Knowledge Graph?". The response of the panelists varied from some structured graph that helps the applications to something that has clean and formal semantics whose domain should be modeled in Web Ontology Language (OWL), which is a fragment of first order logic. All the panel members agreed that knowledge graphs should have some form of semantics and that semantics play a crucial role in differentiating a knowledge graph from other structured sources such as taxonomies. For the question on the available tools and their maturity, all the panel members agreed that although there are good number of tools that are available, more tools are required to increase the uptake of semantic technologies and the tools should provide good documentation to help the users in quickly adopting them. The panel discussion ended with an audience question on whether knowledge graphs have reached the inflection point on the hype curve. Panel members remarked that although the name keeps changing (Knowledge Graphs, Linked Data, RDF, OWL, Frames), the problems that it helps tackle and the issues that are yet to be solved remain. So perhaps the name of "Knowledge Graph" might change in a few years, but the general theme of having semantic structures with domain knowledge helping applications make better decisions would continue.

We would like to thank all the authors for their submissions and presentations at the workshop. We are very grateful to our keynote and invited speakers for their inspiring talks and the program committee members for helping us with their timely reviews. We thank the organizers and the workshop chairs at the Web Science Conference, Fabrizio Silvestri, Matthew Weber and Kathy Fontaine for their support. Thanks to all the participants 
for lively discussions. We would also like to thank Kareem Aggour from GE Global Research and Prof. Tim Finin from the University of Maryland, Baltimore County for their valuable suggestions. Further details of the workshop can be found here: https: //industrial-knowledge-graphs.github.io/2017/.

\section{CHAIRS}

Varish Mulwad, GE Global Research, USA

Raghava Mutharaju, GE Global Research, USA

\section{PROGRAM COMMITTEE}

Adila Krisnadhi, Wright State University, USA

Alfredo Gabaldon, GE Global Research, USA

Craig Knoblock, University of Southern California, USA

Freddy Lécué, Accenture Technology Labs, Ireland

Geeth R De Mel, IBM Research Center, UK

Geetha Manjunath, NIRAMAI, India

Jay Pujara, University of California, Santa Cruz, USA

Jennifer Sleeman, University of Maryland, Baltimore County, USA

Pavan Kapanipathi, IBM T.J Watson Research Center, USA

Prajit Das, University of Maryland, Baltimore County, USA

Sören Auer, University of Bonn, Germany

Steve Gustafson, Maana, USA

Zareen Syed, IBM T.J Watson Research Center, USA 